\title{
Sex and Diabetes: A Thematic Analysis of Gay and Bisexual Men's Accounts
}

\author{
Adam Jowett ${ }^{1}$, Elizabeth Peel and Rachel L. Shaw
}

Please cite the published version of this paper available from the publisher's website: http://hpq.sagepub.com/content/17/3/409.abstract

Jowett, A., Peel, E., \& Shaw, R.L. (2012). Sex and diabetes : a thematic analysis of gay and bisexual men's accounts. Journal of health psychology, 17 (3), 409-418.

DOI: $10.1177 / 1359105311412838$

\begin{abstract}
Around 50 per cent of men with diabetes experience erectile dysfunction. Much of the literature focuses on quality of life measures with heterosexual men in monogamous relationships. This study explores gay and bisexual men's experiences of sex and diabetes. Thirteen interviews were analysed and three themes identified: erectile problems; other 'physical' problems; and disclosing diabetes to sexual partners. Findings highlight a range of sexual problems experienced by non-heterosexual men and the significance of the cultural and relational context in which they are situated. The personalized care promised by the UK government should acknowledge the diversity of sexual practices which might be affected by diabetes.
\end{abstract}

Keywords

diabetes, diversity, men's health, qualitative methods, sexual health, sexuality

1 Corresponding author:

Dr Adam Jowett,

Department of Psychology \& Behavioural Sciences,

Coventry University,

CV1 5FB, UK.

Email: Adam.Jowett@coventry.ac.uk 


\section{INTRODUCTION}

Diabetes is a group of disorders that interferes with the body's ability to use glucose in the blood properly and affects over two million people in England alone (Department of Health (DoH), 2010). Approximately 15 per cent of these have type 1 which is unpreventable and often diagnosed in childhood, while type 2 is the more common form and is strongly associated with 'lifestyle' factors such as obesity (DoH, 2010). If not managed well both forms of diabetes can lead to a range of acute, chronic and life-threatening complications. Those requiring daily insulin injections must administer their dosage carefully in order to avoid the acute complication of hypoglycaemia (low blood sugar). ${ }^{1}$ More chronic complications result from prolonged elevated blood glucose levels and include renal failure and stroke as well as other life changing complications such as blindness, gastrointestinal problems and sexual difficulties.

Diabetes UK (2007) suggests that a discussion of sexual problems such as erectile dysfunction (ED) should be considered part of any diabetes annual review as it can be a symptom of damage to nerves and blood vessels. Fedele et al. (2000) have shown that around half of men who have diabetes experience ED, while Rance et al. (2003) found that men identified ED as the third most concerning complication of diabetes after kidney disease and blindness (Rance et al., 2003). In short, ED is an important issue for men which has not been given due attention. There is limited research about sexual dysfunction among those with diabetes, and that which is available does not consider possible differences between experiences and needs of gay and bisexual men compared to their heterosexual counterparts.

Very little research has explored the range of sexual difficulties related to diabetes and the socio-cultural and relational contexts in which they are experienced. The few studies that have begun to examine this issue have largely taken the form of quality of life assessments and attempts to measure 'sexual bother' (e.g. De Berardis et al., 2002; Penson et al., 2003, 2009). For instance, diabetic men with ED report significantly poorer quality of life as a result of ED (De Berardis et al., 2002). However, these studies tell us little about the men's own experience of ED in a meaningful way. Such research typically applies quality of life scales to measure the psychological impact of biomedically defined sexual problems without opportunity to discuss other issues related to sexuality and diabetes. The very definition of ED is often, either explicitly or implicitly, framed in terms that are inappropriate for gay men. For example, a self-help text by the American Diabetes Association (Roszler and Rice, 2007: 8) poses the following question: 'Have you been experiencing difficulty recently in achieving erections that you and your partner consider adequate for vaginal intercourse?' Although one could argue that sexual problems such as ED are the same for gay and heterosexual men, an erection adequate for vaginal intercourse may differ from that needed for anal or oral penetration (Goldstone, 1999) and insertive and receptive roles are potentially reversible in sex between men (Sandfort and De Keizer, 2001). Also if we 
look beyond the physical, to the psychosocial and cultural, the differences may be greater still.

The vast majority of participants in studies about diabetes and ED are (heterosexually) married and in monogamous relationships (e.g. LeMone, 1993; Penson et al., 2003, 2009). However, expectations and concerns about sex may differ between heterosexual and gay men, and the experience of sexual difficulties within an exclusive relationship may differ to that of casual encounters (Bancroft et al., 2005). In short, sexual problems occur within a social, relational and cultural context which is fundamental to adequate and appropriate support provision (Campbell and Whiteley, 2006). In the present study we address the absence of non-heterosexual men within the literature by looking specifically at accounts from gay and bisexual men with diabetes about their sexual experiences.

\section{METHOD}

Thirteen men with diabetes were interviewed about a range of issues relating to diabetes in their everyday life including how they came to be diagnosed, their management of the condition and how diabetes affected their personal relationships. Although type 1 and type 2 diabetes are considered quite different clinically, many of the complications such as sexual dysfunction and their psychosocial implications may be similar and therefore were considered together in this study. As Campbell et al. (2003: 674) suggest, qualitative health research 'should not be driven by medical considerations but should rather concern itself with the way in which patients' experience disease and illness'. See Table 1 for participants' demographic details.

Table 1. Participant information.

\begin{tabular}{|l|l|l|l|l|l|l|}
\hline Pseudonym & Age & $\begin{array}{l}\text { Sexual } \\
\text { identity }\end{array}$ & $\begin{array}{l}\text { Diabetes } \\
\text { type }\end{array}$ & $\begin{array}{l}\text { Duration } \\
\text { since } \\
\text { diagnosis }\end{array}$ & $\begin{array}{l}\text { Relationship } \\
\text { status }\end{array}$ & $\begin{array}{l}\text { Country of } \\
\text { residence }\end{array}$ \\
\hline Alistair & 59 & Gay & 2 & 3 years & Single & UK \\
\hline Gordon & 69 & Gay & 1 & 43 years & In a relationship & UK \\
\hline Colin & 62 & Gay & 1 & 40 years & Single & UK \\
\hline Enzo & 47 & Gay & 2 & 8 years & Single & UK \\
\hline Ben & 28 & Gay & 1 & 8 years & In a relationship & USA \\
\hline Justin & 41 & Gay & 1 & 35 years & Single & USA \\
\hline John & 43 & Bisexual & 1 & 39 years & Single & USA \\
\hline Graham & 39 & Gay & 1 & 29 years & In a relationship & UK \\
\hline
\end{tabular}


All US participants and one UK participant were recruited through a previous online survey about lesbian, gay and bisexual people's experiences of chronic illness (Jowett and Peel, 2009). The remainder were recruited via an advert in Balance, a Diabetes UK magazine. Although we acknowledge that different locations and health care systems have implications for patients' experiences, there are similar patterns of treatment for diabetes in both countries. The key difference is access; universal access to care is provided by the UK National Health Service (NHS) while a market-based system operates in the USA (Mainous et al., 2006).

Following university ethical approval and informed consent from participants, interviews were conducted by the first author in 2008-2009. US participants were interviewed online via instant messaging. Because of the protracted nature of online interviews (Jowett et al., in press) they lasted approximately three hours with the opportunity for breaks. Face-toface interviews lasted one to two hours, were audio-recorded and transcribed verbatim. Participants were told they could refuse to answer any question, ask to end the interview at any time or withdraw their data retrospectively. We explained how their data would be used and that all would be assigned a pseudonym.

Data were analysed using thematic analysis (Braun and Clarke, 2006). Themes were identified which related to sexual difficulties associated with diabetes. The themes identified were 'problem-based' and within each theme we paid particular attention to the meaning given to the problem, as well as the socio-cultural and relational context in which the problem was situated. Our analysis takes a critical realist approach (Willig, 1999) whereby meaning is understood as constructed through language; however material reality is viewed as constraining the range of possible subject positions available for the individual. We viewed this as an ethical position, as it affirms the embodied reality of living with diabetes while recognizing that these accounts are socially constructed. We have also sought to move away from the medically defined concept of 'sexual dysfunction' adopting instead the broader, more socially circumscribed concept of 'sexual difficulty' (Hurley and Prestage, 2007; Richters et al., 2003).

\section{FINDINGS}

Three problem-based themes were identified: erectile problems; other 'physical' problems (thrush and hypoglycaemia); and disclosing diabetes to sexual partners.

\section{Erectile problems}

Erectile problems were the most commonly mentioned sexual difficulty among participants. While all were aware that erectile difficulties were a possible complication of diabetes, these men found it difficult to determine whether their own poor erections were caused by other factors such as age or stress. The perceived severity and psychosocial impact of such difficulties varied between the men with many describing the problem as 'manageable' with medication. None of the US participants suggested a lack of health insurance prevented them 
access to medication but several UK participants suggested the quantity of oral prescription drugs available on the NHS for ED is inadequate for an active sex life. For others, erectile problems had a more profound impact on their life:

It ['impotence'] had over the years killed my social life ... I can't just chat to somebody in a pub ... I remember on one occasion I went into a gay pub and there was a lad l'd been eyeing up for months, stunning from my point of view and erm... next thing I knew he was standing next to me. I couldn't speak, I could not speak and he came to stand next to me, somebody he knew fancied him. I gulped it down and ran out the club. I can't believe I did that now but I couldn't even talk to him. I was too frightened because I wouldn't want somebody on our local scene to know that erm, I couldn't rise to the occasion. And that's the one problem with gay life ... in heterosexuality, you meet on another occasion and you go out and you go for dinner or for whatever and you chat to them. It's a long time before you actually got into bed, but the gay life was never like that, that was almost always the first time you meet. So that was terrifying me I couldn't do it. (Colin)

In this extract, Colin's account is contextualized by his identity as a gay man, attending commercial venues on a gay 'scene' and belonging to a culture which follows different relationship 'rules' than heterosexual culture (cf. Mutchler, 2000). Before the interview, Colin commented that he did not consider himself a 'typical gay man' as he had had little sex during his life, which he attributed to the lack of confidence described here. The account above also suggests that fear of embarrassment has prevented Colin forming relationships, invoking the idea of gossip spreading throughout his small local gay community.

Colin also invokes the idea of two distinct and dichotomous cultures; 'gay life' in which gay men engage in sexual activity quickly after meeting a partner and 'straight life' whereby sex is preceded by a courting process. This context of sexualized norms within gay culture was described as shaping these men's experience of sexual difficulties related to diabetes:

It can cause problems because, as a gay guy, obviously a major part of the gay culture is basically sex, y'know, it's very promiscuous, very sexually orientated, very physically orientated and if you suffer from impotence - if you're 70 odd then they don't care, but when I was in my 30s, when I was in my early 30s and I'm going what!? Well if you go to a bath house, like a gay sauna, y'know it can definitely be erm, not so much the physical side, but it stresses your - self-worth, you lose your sense of self-worth. You lose your confidence, it's a confidence thing. (Enzo)

The sexualized norms of the communities to which they belong were framed as highly problematic for these men, shaping the way they viewed themselves. Having sex thus 
becomes integral to the maintenance of a gay or bisexual identity (Braun et al., 2009; Mutchler, 2000). Above, Enzo suggests that experiencing erectile problems when attending gay saunas has resulted in a loss of confidence and self-worth. Although he does not specify what exactly it is about this environment that makes such problems particularly difficult, the anonymous and depersonalized nature of sex-on-site venues may not be sensitive to the emotional needs of men who experience sexual difficulties (Haubrich et al., 2004).

While ED is generally defined in medical discourse as an inability to achieve or maintain an erection adequate for (assumed-to-be-vaginal) intercourse (Steidle, 2002), some of these men stated that they did not engage in anal intercourse at all, nor did they desire to do so: 'I've never given or received anal sex. I never wanted to' (Colin). Yet, erectile problems were spoken of in the context of other sexual activities:

If it's a one night stand then yeah even if it's only a hand job by them, you want a reason for it, and they might think that they don't turn me on, y'know, and that would be rotten for me, if I really was attracted to them physically then I don't want it to look as if I wasn't. So again it's embarrassment I suppose in a way, but yeah l'd lose them for that reason. (Colin)

This extract highlights that the meanings ascribed to erections are wider than the medical discourse of sexual 'function' implies. Within a medical model, the 'function' of an erection is its ability to penetrate and engage in intercourse. In contrast, it is the psychosocial impact these men emphasize; the embarrassment experienced and fear of losing a potential partner An erection can also be viewed as communicating sexual arousal and attraction to a partner. Also, despite ED often being defined as an inability to engage in intercourse, Colin suggested that he had only ever used his prescribed medication for masturbation. Similarly Enzo also spoke about his erectile problems in relation to masturbation:

The only thing I resent, if you said to me 'is there anything you resent about diabetes?' it's erm because I'm a guy, I do things that guys do when they're on their own, and the mechanics don't work. There's many a time I lie in bed and it doesn't matter how hard I try, I'm on my own, and my body just will not co-operate and that gets me down. (Enzo)

Enzo's experience illustrates that ED is not solely a problem relating to 'performing' for a partner but is about his relationship with his own body and frustration with its non-cooperation. Just as the ability to have sex was part of Enzo's perception of being within the norms of the gay community, the ability to masturbate is framed here as part of his identity as a man. So rather than ED representing an impediment to the 'natural' function of penetrative intercourse, as the medical model suggests, within these accounts erectile difficulties were 
interwoven with and problematic for these participants' identities as men and in particular as gay or bisexual men.

\section{Other 'physical' problems}

While erectile problems are typically the sole sexual problem addressed within the diabetes literature, these men reported a number of other more acute complications of their condition which affected their sex lives. For example, in the extract below, Ben describes his experience of thrush, and its impact on sexual activity between him and his partner:

Ben: As a diabetic, I sometimes suffer from a Candidiasis in my buttocks. This makes it impossible to be the 'bottom' in anal intercourse.

Adam: Ok. And is that a big issue for you? I mean do you adjust what you do in the bedroom because of that?

Ben: It hasn't been all that much of a concern. I do prefer to top and my partner is usually willing to bottom, but there are times we'd like to switch and haven't been able to ... there have been two or three occasions in which a particularly bad Candidiasis infection has caused an odor which makes oral sex unpleasant. In those situations, my partner and I have been limited to mutual masturbation or have abstained from sexual contact altogether.

Although thrush is commonly reported as a problem for women with diabetes (e.g. Roszler and Rice, 2007), candida infections in the anogenital region are also common among men with diabetes, which may be particularly problematic for gay or bisexual men who engage in receptive anal intercourse or anal-oral sex ('rimming') (Goldstone, 1999). The above extract highlights the flexibility in sex between men meaning that gay or bisexual men experiencing ED may choose to be the receptive partner in intercourse, which does not necessitate an erection (Bancroft et al., 2005). However, such issues may not be being reported to general physicians; for example Ben reported that he was not 'out' to his endocrinologist and visited a specialist sexual health clinic for gay men when being screened for sexually transmitted diseases (STD):

I feel that l'd have to discuss any sexual problems related to my diabetes with my endochrinologist. ${ }^{2}$ I did have discussed the Candidiasis with him, but I did not disclose its affect on my sex life. If the impotence problems continue, I will also discuss those concerns with him. The gay health clinic in [name of city] seems to operate exclusively for the testing of STDs and the counseling of those who find themselves testing positive for an STD ... I have considered exchanging the care of my endochrinologist for the care of an internal medicine specialist in [name of city] who advertises as gay-friendly. If diabetes ever had a significant enough impact on my sex life as a gay man, then I almost certainly would make that change. (Ben) 
Gay and bisexual men may be wary of discussing their sexual behaviour with health professionals, not least because of the historical medicalization of gay men's sexuality (Scarce, 2000). However, while gay and bisexual men may wish to limit unwanted medical surveillance of their sexual behaviour, it is important that they have access to health services that allow for a discussion of such problems. Ben's admission that if diabetes had a significant impact on his sex life as a gay man he would exchange his care to someone who advertised as 'gay friendly', suggests a degree of trepidation about discussing sex with his current physician. Gay and bisexual men may be reluctant to disclose their sexual identity to health professionals for fear of potential repercussions to the quality of care they receive (Jowett and Peel, 2009; Stein and Bonuck, 2001). Riggs (2009) suggests that heterosexism operates subtly in this way. The mere possibility that discrimination may occur has the power to influence gay and bisexual men's decisions to not be 'out' in certain environments and thus influence what they choose to discuss in a medical consultation. The compartmentalization of Ben's health care between the 'gay health clinic' and his endocrinologist could become problematic in terms of continuity of care. Indeed the separation of his diabetes care and his sexual health creates a situation in which Ben may feel unable to discuss sexual difficulties related to diabetes with either.

Another problem reported by these participants was experiencing hypoglycaemia during, or resulting from, sex. Symptoms of 'hypos' can vary widely including shakiness, sweating, nausea, difficulty speaking and behaviour likened to drunkenness. Having a hypo was commonly described as interrupting sexual activity by causing ED or by having to get something to eat or drink in order to raise their blood glucose. It was also described as requiring explanation:

It affects your sex life radically. Erm, there are times when if it all gets too energetic at the end of it I get the shakes or I'm half way to collapsing and the other guy who may have come to meet me for the first time, it's gonna scare the shit out of em, because they're wondering what the hell they've walked into, y'know what I mean? So what I do now is, I deliberately overload my body. I eat an entire packet of biscuits if I know somebody is coming, to counter balance y'know what I mean. (Enzo)

I've had bad hypos in the middle of the night with them [his partners] in fact one, two, three, I've had hypos with all of them at different times and of course they know about the diabetes and I've warned them about it ... I generally put a lucozade by the bed and say 'listen if I get into trouble there's the lucozade alright' ... it's difficult for boyfriends, it's so unexpected and they've never seen it before. (Gordon)

We see here how Enzo deliberately maintains a high blood glucose level before sex in order to avert such a situation occurring. In contrast, Gordon explained how he prepared for this 
eventuality by warning partners, keeping energy drinks containing glucose nearby and explaining to partners what to do. The different relational contexts of these two accounts may influence the strategies described here. Enzo describes a situation in which someone is potentially meeting him for the first time, while Gordon refers to partners who know about his diabetes. Indeed, communication about diabetes with new or casual partners was a common theme in the men's talk; one which we consider a 'difficulty' in itself.

\section{Disclosing diabetes to sexual partners}

Many of the men spoke about whether or when they would tell new sexual partners they had diabetes. This was described as a particular dilemma for those using an insulin pump. The infusion site's location on the body, together with the relational context of the sexual encounter was described as influencing decisions regarding whether or not to offer an explanation:

I'm single and when I meet up with guys, I'm the proverbial queer and like my sex. But being on an insulin pump, I usually end up telling them what the infusion site is all about 'cause it does look odd especially when it's an odd spot like my inner thigh or near a nipple on my chest ... I just don't like guys wondering about it when they inevitably see it. I'm usually unhooked from pump, so it's just the site. I tell them that I'm a diabetic and on an insulin pump and that is where I hook up to it at. Sometimes it's before, sometimes after. But unless it's just a quickie thing, I tell them 'cause I usually don't like to be unhooked from pump for too long. Anonymous stuff, I don't bother unless they ask ... from guys seeing [the] site before I tell them it's like 'oh man, what's that?!?!?' or 'what's wrong with you, you aren't sick or something are ya?' which I take to mean HIV status and then explain. (Justin)

it's usually a shock to that person ... I always wonder whether people are worried about whether it's some sort of intravenous HIV treatment. (John)

Explaining an insulin pump or its infusion site to a new sexual partner is not a unique experience for a gay or bisexual man but the assumption that partners will think it is HIV related is. This may reflect concerns about another's HIV status before having sex with a new partner generally but is also indicative of the significance of HIV within the psyches of gay and bisexual men. Lipton (2004) has argued that within gay communities notions of illness readily evoke HIV as those with other chronic illnesses are relatively 'invisible' within these communities. Within this context, a direct disclosure of diabetes may be used by some gay and bisexual men as an indirect strategy to pre-empt assumptions and reassure partners regarding their HIV status. ${ }^{3}$

Dilemmas about whether, or when, to tell sexual partners about diabetes were not, however, limited to those using insulin pumps. A number of the men felt that telling new 
partners about their diabetes was a hurdle at the beginning of any new relationship and disclosing this information early on was described as increasing their risk of rejection. For example, Graham stated that when he was single he would avoid staying the night at others' homes to avoid having to inject:

I've probably avoided y'know even staying over and stuff. I probably would have avoided injecting in front of them or - I'd have it with me but I wouldn't because obviously you can't really, because then you've got to open up the whole thing so ... again you just wouldn't because it's kind of like a one night stand so therefore you're never gonna - I mean that would just be really strange if you sort of started talking about stuff like that. (Graham)

This demonstrates how a discussion of one's health with a casual sex partner is understood as violating norms that govern sexual contexts ('really strange'). This has been well established within sexual health research, which tells us that discussions about HIV status are not usual in casual sex encounters and are regarded as an expression of inappropriate intimacy (Davis, 2002; Green and Sobo, 2000). It is unclear from these men's accounts whether the emphasis in the self- help literature about sex and diabetes on discussing concerns with partners (Roszler and Rice, 2007) is always realistic. Such open communication may be particularly challenging in the context of more casual sex encounters ('anonymous stuff') but may also present a barrier when initiating longer-term relationships which may or may not involve penetrative intercourse.

\section{DISCUSSION}

In this article we have examined the perceived impact of diabetes on the sex lives of gay and bisexual men. In line with the high prevalence of ED among men with diabetes, problems getting or maintaining erections were the most commonly reported sexual problem described by this sample. Much of the literature regarding sex and diabetes takes its cue from the medical model of sexuality and sexual 'dysfunction'. As Potts et al. (2004) argue, this medical model homogenizes a diversity of sexual difficulties and experiences, positioning (vaginal) penetrative intercourse as central to all sexual relationships.

In line with Potts et al.'s findings however, the accounts of these gay and bisexual men demonstrate a range of psychosocial and cultural significances attached to erections, and erectile problems were spoken of as more than a physiological impediment to intercourse. Such accounts do not map onto the 'typical' definitions of 'erectile dysfunction'.

In addition to erectile problems, these men spoke of acute symptoms such as candidiasis and hypos as interrupting their sexual activity and described negotiating the disclosure of diabetes with sexual partners. Such problems were described as more complicated in the context of casual sex or sex with new partners who may not be aware of their diabetes. We are not suggesting that these problems are unique to gay or bisexual men. 
The accounts of sexual difficulties analysed here are relevant to all men with diabetes, however, our study does signal some of the ways in which these sexual problems may be experienced differently by gay and bisexual men.

Within the study gay culture was routinely constructed as highly sexualized, which might reflect dominant discourses about gay culture rather than an accurate description of the diverse contexts in which gay and bisexual men live (Braun et al., 2009). As Flowers and Langdridge (2007: 680) suggest 'gay men enjoy a plurality of very different kinds of sexual conduct and both consume and produce very different kinds of sexual cultures'. Nevertheless, gay and bisexual men who feel unable to live up to such stereotypes of gay and bisexual men's sexuality due to chronic illness may experience considerable anxiety (Hanjorgiris et al., 2004). Our analysis also highlights the significance of living with a chronic illness such as diabetes in the context of a community whose notions of illness are consumed by HIV (Lipton, 2004).

Issues such as these should be considered when providing support and counselling for gay and bisexual men living with sexual complications of chronic illness. As Bokhour et al. (2001: 655) comment in relation to their research about prostate cancer and erectile dysfunction, clinicians 'need to probe beyond the mechanics of erectile function, and ask questions about men's feelings about their sexual lives and relationships'. It is useful for health professionals and psychologists to be aware of and appreciate the range of ways in which sexual difficulties may be experienced and their emotional impact. Our findings also have wider implications for health psychology, as within psychological research about health generally, heterosexuality often continues to be the assumed norm (see Boyle, 1993; Peel and Thomson, 2009 for further discussion of heteronormativity in health psychology).

The duel location of participants within the research should be noted. In particular the concerns over discussing sex with and being 'out' to health professionals presented here were voiced by a participant from the USA. There are of course differences between the USA and the UK in terms of legal protection from discrimination granted by their respective governments. Although discrimination on the basis of sexual orientation has been outlawed in the UK, fears of heterosexism and enduring inequalities within health care persist (Jowett and Peel, 2009).

In sum, we have highlighted the dearth of social scientific literature on the topic of sex and diabetes; clearly further research with lesbian, gay, bisexual and trans populations is required. Our findings demonstrate the need for a contextually sensitive approach to sex and diabetes that explores the experiences of a wider range of sexual difficulties within their cultural and interpersonal contexts. They also suggest that awareness training for health professionals may be worth pursuing. Tackling fears of heterosexism by patients is complex and, among other things, is probably dependent on a shift in culture within health care services. Our study further emphasizes the need to look beyond the problems of heterosexually married men, and explicitly recognize a diversity of sexualities. 


\section{NOTES}

1. This includes most people diagnosed with type 1 (unless using other intravenous devices such as an insulin pump) and those with type 2 for whom dietary management and oral medication alone can no longer maintain blood glucose control.

2. Spelling errors from transcripts of online interviews have not been corrected.

3. Of course having diabetes does not preclude the possibility of also being HIV positive. In fact the use of antiretroviral therapies is associated with an increased risk of developing diabetes (Brown et al., 2005).

\section{ACKNOWLEDGEMENTS}

We would like to thank Adam Bourne for his helpful comments on an earlier draft of this article and the men who took part in the study for sharing their stories and speaking so candidly about such a sensitive topic.

\section{REFERENCES}

Bancroft J, Carnes L, Janssen E, Goodrich D and Long SJ (2005) Erectile and ejaculatory problems in gay and heterosexual men. Archives of Sexual Behaviour 34(3): 285297.

Bokhour BG, Clark JA, Inui TS, Silliman RA and Talcott JA (2001) Sexuality after treatment for early prostate cancer. Journal of General Internal Medicine 16(10): 649-655.

Boyle M (1993) Sexual dysfunction or heterosexual dysfunction? Feminism \& Psychology 3(1): 73-88.

Braun V and Clarke V (2006) Using thematic analysis in psychology. Qualitative Research in Psychology 3(2): 77-101.

Braun V, Terry G, Gavey N and Fenaughty J (2009) 'Risk' and sexual coercion among gay and bisexual men in Aotearoa/New Zealand - key informant accounts. Culture, Health \& Sexuality 11(2): 111-124.

Brown TT, Cole SR, Li X, et al. (2005) Antiretroviral therapy and the prevalence and incidence of diabetes mellitus in the multicenter AIDS cohort study. Archives of Internal Medicine 165(21): 1179-1184.

Campbell R, Pound P, Pope C, et al. (2003) Evaluating metaethnography: A synthesis of qualitative research on lay experiences of diabetes and diabetes care. Social Science \& Medicine 56(4): 671-684.

Campbell T and Whiteley C (2006) Working clinically with gay men with sexual performance problems. Sexual and Relationship Therapy 21(4): 419-428.

Davis MDM (2002) HIV prevention rationalities and serostatus in the risk narratives of gay men. Sexualities 5(3): 281-299.

De Berardis G, Franciosi M, Belfiglio M, et al. (2002) Erectile dysfunction and quality of life in type 2 diabetic patients: A serious problem too often overlooked. Diabetes Care 25(2): 284-291. 
Department of Health (DoH) (2010) Six Years On - Delivering the Diabetes National Service Framework (Report No. 301316). London: Produced by COI for the Department of Health. Available at:

http://www.dh.gov.uk/en/Publicationsandstatistics/Publications/PublicationsPolicyAnd Guidance/DH_112509.

Diabetes UK (2007) Sex and Diabetes: A Guide to Sexual Dysfunction. London: Diabetes UK.

Fedele D, Bortolotti A, Coscelli C, et al. (2000) Erectile dysfunction in type 1 and type 2 diabetics in Italy. International Journal of Epidemiology 29(3): 524-531.

Flowers P and Langdridge D (2007) Offending the other: Deconstructing narratives of deviance and pathology. British Journal of Social Psychology 46(3): 679-690.

Goldstone SE (1999) The Ins and Outs of Gay Sex: A Medical Handbook for Men. New York: Dell Publishing.

Green G and Sobo EJ (2000) The Endangered Self: Managing the Social Risks of HIV. London: Routledge.

Hanjorgiris WF, Rath JF and O'Neill JH (2004) Gay men living with chronic illness or disability: A sociocultural, minority group perspective on mental health. In: Lipton B (ed.) Gay Men Living with Chronic IIInesses and Disabilities: From Crisis to Crossroads. New York: Harrington Park Press, 25-41.

Haubrich DJ, Myers T, Calzavara L, Ryder K and Medved W (2004) Gay and bisexual men's experiences of bathhouse culture and sex: 'Looking for love in all the wrong places'. Culture, Health \& Sexuality 6(1): 19-29.

Hurley M and Prestage G (2007) Intensive sex partying: Contextual aspects of 'sexual dysfunction'. Journal of HIV Therapy 12(2): 44-48.

Jowett A and Peel E (2009) Chronic illness in non-heterosexual contexts: An online survey of experiences. Feminism \& Psychology 19(4): 454-474.

Jowett A, Peel E and Shaw RL (in press) Online interviewing in psychology: Reflections on the process. Qualitative Research in Psychology.

LeMone P (1993) Human sexuality in adults with insulin-dependent diabetes mellitus. Journal of Nursing Scholarship 25(2): 102-105.

Lipton B (2004) Gay men living with non-HIV chronic illnesses. In: Lipton B (ed.) Gay Men Living with Chronic IIInesses and Disabilities: From Crisis to Crossroads. New York: Harrington Park Press, 1-23.

Mainous AG, III, Diaz VA, Saxena S, et al. (2006) Diabetes management in the USA and England: Comparative analysis of national surveys. Journal of the Royal Society of Medicine 99(9): 463-469.

Mutchler MG (2000) Young gay men's stories in the States: Scripts, sex, and safety in the time of AIDS. Sexualities 3(1): 31-54.

Peel E and Thomson M (2009) Lesbian, gay, bisexual, trans and queer health psychology: Historical development and future possibilities. Feminism \& Psychology 19(4): 427436. 
Penson DF, Latini DM, Lubeck DP, Wallace KL, Henning JM and Lue TF (2003) Do impotent men with diabetes have more severe erectile dysfunction and worse quality of life than the general population of impotent patients? Diabetes Care 26(4): 1093-1099.

Penson DF, Wessells H, Cleary $\mathrm{P}$, et al. (2009) Sexual dysfunction and symptom impact in men with long-standing type 1 diabetes in the DCCT/EDIC cohort. Journal of Sexual Medicine 6(7): 1969-1978.

Potts A, Grace V, Gavey N and Vares T (2004) 'Viagra stories': Challenging 'erectile dysfunction'. Social Science \& Medicine 59(3): 489-499.

Rance J, Phillips C, Davies S, O'Malley B, Zaman Q and Price D (2003) How much of a priority is treating erectile dysfunction? A study of patients' perceptions. Diabetic Medicine 20(3): 232-238.

Richters J, Grulich AE, De Visser RO, Smith AMA and Rissel CE (2003) Sex in Australia: Sexual difficulties in a representative sample of adults. Australian and New Zealand Journal of Public Health 27(2): 164-170.

Riggs D (2009) The health and well-being implications of emotion work undertaken by gay sperm donors. Feminism \& Psychology 19(4): 517-533.

Roszler J and Rice D (2007) Sex and Diabetes: For Him and for Her. New York: American Diabetes Association.

Sandfort TGM and Keizer M (2001) Sexual problems in gay men: An overview of the empirical research. Annual Review of Sexual Research 12: 93-120.

Scarce M (2000) The second wave of the gay men's health movement: Medicalization and cooptation as pitfalls of progress. Journal of the Gay and Lesbian Medical Association 4(1): 3-4.

Steidle CP (2002) Sexual dysfunction: Male and female issues. International Journal of Fertility 47(1): 32-36.

Stein GL and Bonuck KA (2001) Physician-patient relationships among the gay and lesbian community. Journal of the Gay and Lesbian Medical Association 5(3): 87-93.

Willig C (1999) Beyond appearances: A critical realist approach to social constructionism. In: Nightingale DJ and Cromby J (eds) Social Constructionist Psychology: A Critical Analysis of Theory and Practice. Buckingham/Philadelphia, PA: Open University Press, 37-52. 